\title{
Vorrede zur zweiten Auflage
}

Das vorliegende Werk hat in seiner ersten Auflage eine sehr gute Aufnahme gefunden. Schon innerhalb des ersten Jahres sind I 100 Exemplare abgesetzt worden, und nur die große Auflage, sowie namentlich die Beschäftigung mit anderen medizinischen Problemen haben mich verhindert, bereits früher an die Neubearbeitung des Gebietes heranzutreten.

Nicht verschweigen will ich, daß meinen auf EHRLICH schem Boden stehenden Ansichten auch manche Opposition erwachsen ist. Das erscheint bei dem heftigen Kampf der Ansichten über eine große Zahl von Grundfragen als selbstverständlich.

Ich habe mich auch in der Neubearbeitung bemuiht, gegnerische Auffassungen, so viel es der Raum gestattete, zum Worte kommen zu lassen, und der Darstellung eine weitgehende Objektivität einzuprägen. Dennoch muß nach meiner Auffassung die eigene persönliche Ansicht beständig die Leitung übernehmen, wenn das Buch einen Wert beanspruchen darf. So verlangen ja z. B. selbst heute Werke von referierendem Charakter, wie die Ergebnisse der innern Medizin und Kinderheilkunde, von den Autoren eine Darstellung nach persönlicher Auffassung.

Der zweiten Auflage sind eine außerordentlich große Zahl von eigenen klinischen Beobachtungen und histologischen Untersuchungen zugrunde gelegt. Rein äußerlich zeigt sich das schon in der Vermehrung der Tafeln und des Inhaltes. Desgleichen hat die Literatur, deren Strom auf diesem Gebiete mit unverminderter Kraft daherfließt, eine sehr starke Berücksichtigung gefunden. Für einen Teil der fremdsprachigen Erscheinungen war das nur möglich durch die ausgezeichneten Referate der Folia haematologica, was ich gerne anerkenne; denn schon heute wäre 
es niemandem mehr möglich, selbst auf diesem beschränkten Gebiete alles in Originalen einzusehen.

Vor allem ist in der Neuauflage die eingehendste Darstellung der Morphologie zur Geltung gekommen und wie ich annehmen zu dürfen glaube, in einem Umfang, den keine andere Bearbeitung dieses Gebietes erreicht. Es erschien mir eine so weit gehende Berücksichtigung morphologischer Fragen durchaus nötig, geben sie uns doch die Basis für die Auffassung und Kritik vieler klinischer Verhältnisse.

Ich hoffe, daß meine stetige und intensive Beschäftigung mit den Naturwissenschaften, speziell mit systematischer Botanik, mich in der Beobachtung und ganz besonders in der Bewertung morphologischer Befunde gefördert hat; handelt es sich doch in der Histologie wie in den Naturwissenschaften um die gleichen Prinzipien, um die Abstraktion allgemeiner Gesichtspunkte aus der Vielheit und Variation der äußeren Formen.

Gleichwohl sind aber auch die physikalischen Methoden sehr stark berücksichtigt worden, so beispielsweise die Viskosimetrie, der ich in Verbindung mit der Morphologie einen wichtigen Platz in der klinischen Blutuntersuchung vindiziere.

Im wesentlichen sind es aber auch in der Neuauflage biologisch-klinische Gesichtspunkte, welche auf der Basis eingehendster Morphologie in inniger Verbindung mit pathologischer Anatomie und in Berücksichtigung experimenteller und embryologischer Forschungen den Grundplan dieses Werkes gelegt haben.

Trotz aller Hochschätzung der Morphologie erscheint es mir $z$ weifellos, daß in vielen hier erörterten Problemen die reine morphologische Untersuchung nicht zu sichern Ergebnissen fürt, da ja verschiedene Forscher bei gleichen oder doch wenig abweichenden Befunden zu ganz verschiedenen Deutungen und Schlüssen kommen. Da verlangt die klinisch-biologische Forschungsrichtung mit Recht ihre volle Gleichwertigkeit und vermag manche Probleme 
einer Lösung näher zu führen, wenn die reine Morphologie uns kein unzweideutiges Ergebnis bietet.

Die stärksten Erweiterungen betreffen den ersten Teil meines Werkes (Untersuchungsmethoden und Histologie), während die klinische Darstellung der Blutkrankheiten keine größeren Änderungen erfordert hat, zumal schon in der ersten Auflage die Kapitel Leukämie und Pseudoleukämie völlig in der heute wohl allgemein anerkannten Auffassung als Systemaffektionen niedergelegt worden sind.

Meinem Freunde, Prof. Dr. ERICH MEyer in Straßburg, verdanke ich die Darstellung des Kapitels der paroxysmalen Hämoglobinurie, auf welchem Gebiete er mit eigenen Forschungen, unser Verständnis des Leidens fördernd, eingegriffen hat.

Ich hoffe, daß auch diese neue Auflage in manchen Fragen der Hämatologie Anregung und Aufklärung bringt und zu der Erweiterung unseres Wissens beiträgt.

Zürich, Oktober I9I I.

O. Naegeli 